\title{
Removal of baseline wandering in ECG signal by improved detrending method
}

\author{
Seung-Won Shin ${ }^{\mathrm{a}}$, Kyeong-Seop Kim ${ }^{\mathrm{b}, *}$, Chul-Gyu Song, ${ }^{\mathrm{c},}$, Jeong-Whan Lee ${ }^{\mathrm{b}}$, Jeong-Hwan \\ Kim $^{\mathrm{b}}$ and Gyeo-Wun Jeung \\ ${ }^{a}$ Biomedical Engineering Branch Division, National Cancer Center, Goyang-si, Gyeonggi-do, Korea \\ ${ }^{b}$ School of Biomedical Engineering, Konkuk University, Chungju-si, Chungbuk, Korea \\ ${ }^{c}$ Electronic Engineering Department, Chonbuk National University, Jeonju-si, Jeonbuk, Korea
}

\begin{abstract}
The very first step to process electrocardiogram (ECG) signal is to eliminate baseline wandering interference that is usually caused by electrode-skin impedance mismatch, motion artifacts due to a patient's body moment or respiratory breathing. A new method is thus suggested to remove baseline wandering in ECG by improving the detrending method that was originally proposed for eliminating slow non-stationary trends from heart rate variability (HRV). In our proposed method, a global trend is estimated in terms of baseline wandering by merging the local trend based on an ECG segment that represents a part of the ECG signal. The experimental results show that the improved detrending method can efficiently resolve baseline wandering without distorting any morphological characteristic embedded in the ECG signal in no time delay manner.
\end{abstract}

Keywords: Electrocardiogram (ECG), baseline wandering, detrending, heart rate variability (HRV), high-pass filter

\section{Introduction}

A number of studies have been conducted to correct the baseline fluctuations under the assumption that the drift wanders within the low-frequency bandwidth below $1 \mathrm{~Hz}$. Van Alste, et al. [1] designed a Finite Impulse Response filter to remove baseline fluctuation and Ezenwa, et al. [2] also eliminated the interference by chasing an equi-potential surface (iso-electric region) with applying the baseline tracking algorithm that utilizes the characteristics of ECG signal. Laguna [3] and Jane, et al. [4] implemented the cascaded adaptive filter in two phases and Pandit [5] devised an algorithm to correct baseline wandering by applying Short-Time Fourier Transform. Shusterman, et al. [6] suggested a selective filter to nullify the effect of baseline wandering. Dotsinsky, et al. [7] applied bi-directional digital filtering algorithm and Mneimneh, et al. [8] exploited the qualities of Kalman filter using an approximated polynomial function that reflected the features of ECG signal. Mateo, et al. [9] considered Many Adaptive Linear Neurons (MADALINE) artificial neural network model to delete

\footnotetext{
* Address for correspondence: Kyeong-Seop Kim, College of Biomedical \& Health Science, School of Biomedical Engineering, Konkuk University, 268 Chungwondaero, Chungju-si, Chungbuk, Korea. Tel.: +82 43840 3765; Fax: +82 43 851 6265; E-mail: kyeong@kku.ac.kr.

Chul-Gyu Song, Dept. of Electronic Eng., Center for Advanced Biomedical Research, Chonbuk National University, 66-14 Deokjin-dong, Jeonju-si, Jeonbuk, Korea. Tel.: +82 63270 4282; Fax: +82 63270 2394; E-mail: song133436@gmail.com.
}

0959-2989/15/\$35.00 @ 2015 - IOS Press and the authors. 
ECG baseline wander. Additionally, Zhao, et al. [10, 11] and Blanco-Velasco, et al. [12] tried to remove baseline fluctuation and simultaneously suppress the additive noise including power line interference in ECG signal by applying empirical mode decomposition and adaptive filter. Iliev, et al. [13] proposed a composite filter to delete both powerline interference and baseline wandering. Rahman, et al. [14] removed the artifacts by applying adaptive filter based on error nonlinearity Least Mean Square (LMS) algorithm. Furthermore, the Constrained Stability Least Mean Square (CSLMS) algorithm [15] was applied to decrease mean-square error of LMS algorithm. The wavelet transform was considered to eliminate baseline wandering in ECG by implementing a search algorithm based on computing wavelet packet coefficients [16] and a hierarchical model utilizing Independent Component Analysis (ICA) [17] was proposed to suppress the baseline wandering in the ECG. To reduce baseline wandering for bioelectrical signals, constrained convex optimization problem [18] was solved and the fractal modeling [19] was also considered by interpreting baseline fluctuation as the first order Brownian-motion process. All of the previously suggested digital filters to reduce baseline wandering face a drawback: a certain amount of time delay is required to get the filtered signal. Thus, the meaningful information contained in the initial stage of getting ECG signal can't be resolved or can be lost due to the failure of correcting baseline distortion. Furthermore, the artificial neural network such as MADALINE model requires a proper training stage to approach the desired output signal and it is difficult to handle the persistent non-stationary trend especially in a long-term ECG signal. Therefore, this paper suggested a new method to remove baseline wandering in ECG by improving the detrending method that was originally proposed for eliminating slow non-stationary trends from Heart Rate Variability (HRV).

\section{Method}

\subsection{Detrending with smoothness priors formulation}

Detrending procedure estimates the trend of the given signal based on smoothness prior formulation. Tarvai, et al. [20] presented a detrending method and tested its properties to remove the fluctuating level of RR intervals in ECG data before HRV analysis was considered.

Eqs. (1)-(6) show the expressions for estimating the trend by detrending procedure [20].

$$
\begin{gathered}
z=z_{\text {stat }}+z_{\text {trend }} \\
z_{\text {trend }}=H \theta+v \\
\hat{z}_{\text {trend }}=H \hat{\theta}_{\lambda} \\
\hat{\theta}_{\lambda}=\left(H^{T} H+\lambda^{2} H^{T} D_{d}{ }^{T} D_{d} H\right)^{-1} H^{T} z \\
D_{d}(d=2)=D_{2}=\left(\begin{array}{cccccc}
1 & -2 & 1 & 0 & \cdots & 0 \\
0 & 1 & -2 & 1 & \ddots & \vdots \\
\vdots & \ddots & \ddots & \ddots & \ddots & 0 \\
0 & \cdots & 0 & 1 & -2 & 1
\end{array}\right) \\
\hat{z}_{\text {stat }}=z-H \hat{\theta}_{\lambda}=\left(I-\left(I+\lambda^{2} D_{2}{ }^{T} D_{2}\right)^{-1}\right) z
\end{gathered}
$$

Where, $z$ is an input signal consisting of the stationary part, $z_{\text {stat }}$ and the low-frequency component, $z_{\text {trend. }} v$ is an observation error and $H$ is an observation matrix. $\theta$ is the regression parameter and $\lambda$ is a 
Table 1

$\lambda$ and the corresponding cutoff frequency

\begin{tabular}{ll}
\hline \hline$\lambda$ & $F_{c}$ \\
\hline 1 & $0.189 \times F_{s}$ \\
2 & $0.132 \times F_{s}$ \\
3 & $0.093 \times F_{s}$ \\
10 & $0.059 \times F_{s}$ \\
20 & $0.041 \times F_{S}$ \\
50 & $0.025 \times F_{s}$ \\
300 & $0.011 \times F_{S}$ \\
\hline \hline
\end{tabular}

regularization parameter for estimating $\hat{\theta}_{\lambda}$ in the least square sense. Thus, $\hat{\theta}_{\lambda}$ denotes the estimated regression parameter by applying a regularization parameter, $\lambda$. $D_{d}$ indicates the difference matrix approximating $d^{\text {th }}$ derivative operator and consequently $D_{2}$ denotes the second order difference matrix. The stationary component $\hat{z}_{\text {stat }}$ can be estimated by subtracting non-stationary trend, $H \hat{\theta}_{\lambda}$.

\subsection{Improved detrending algorithm}

The process for removing the baseline wandering in the ECG signal by detrending method starts from setting the cutoff frequency through the determination of the regularization parameter, $\lambda$ and then removes the non-stationary trend by applying Eq. (6). The optimal value of $\lambda$ can be derived by applying the regression analysis to the data points as stated in Table 1 [20].

Eq. (7) shows a curve fitting result by converting an exponential model into a linear one with the logarithmic transformation.

$$
F_{c}=0.1865 \lambda^{-0.5022} \cdot F_{S}
$$

where $F_{c}$ is the cut-off frequency and $F_{S}$ means the sampling frequency.

In contrast with FIR and IIR digital filter, the entire segment in the input signal is considered during

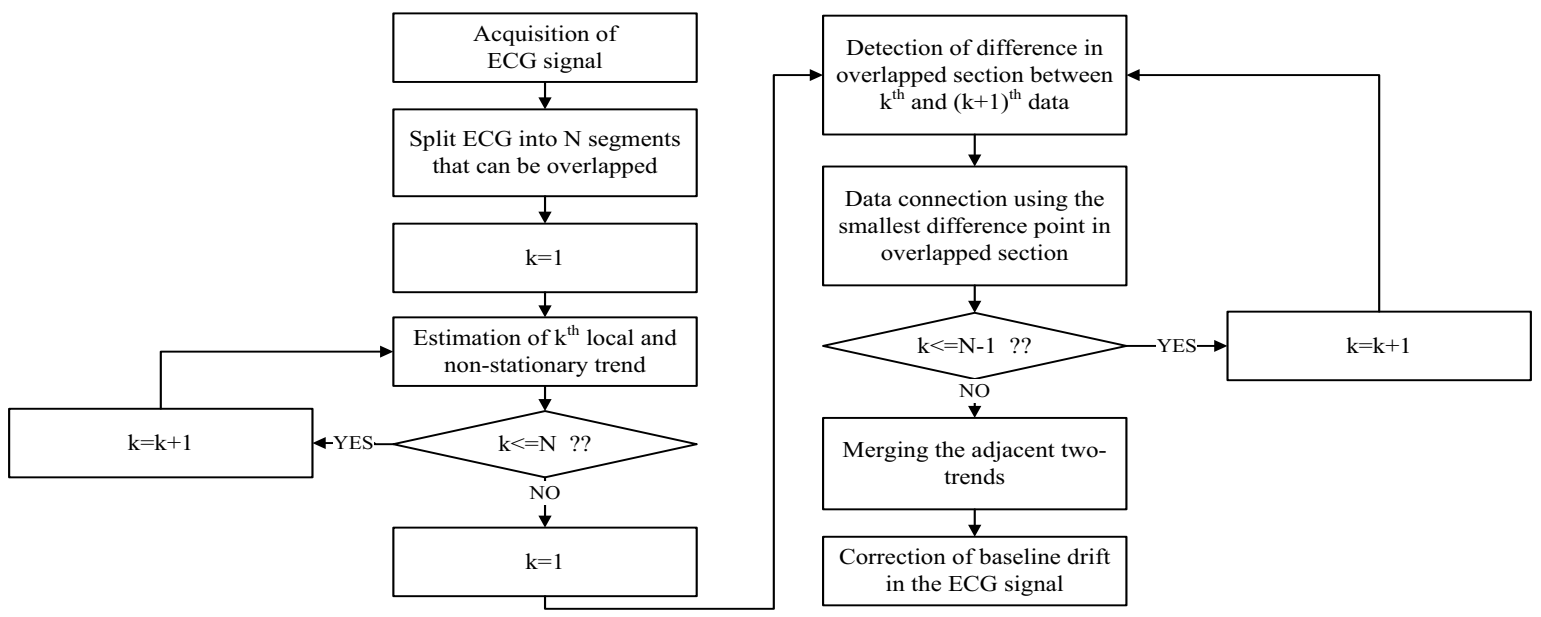

Fig. 1. A block diagram for illustrating our proposed detrending method. 


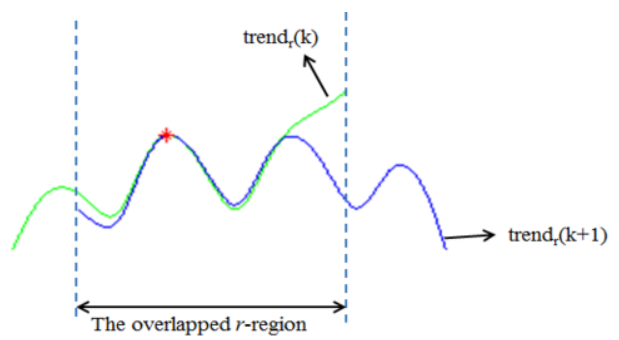

Fig. 2. Merging two-adjacent trends, $\operatorname{trend}_{r}(k)$ and $\operatorname{trend}_{r}(k+1)$

the detrending operation and it has the advantage of no time delay in getting the processed signal. However, due to the requirement of computing the inverse of a matrix, this approach is not applicable to a long-term ECG or a large volume of data. Thus, to overcome this inherent problem for correcting baseline wandering in the ECG signal, a new detrending algorithm is proposed as illustrated in Figure 1 .

Eq. (8) describes the process that connects two adjacent-trends.

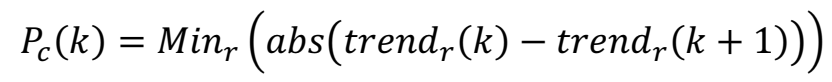

where $P_{c}(k)$ is $k^{\text {th }}$ connecting point, $r$ is the overlapped region and $\operatorname{trend}_{r}(k)$ is the overlapped section of $k^{\text {th }}$ trend data. Figure 2 shows the merging process by connecting two-adjacent trends in the overlapped $r$-region with identifying $P_{c}(k)$ to guarantee a smooth connection between two trends.

\section{Results}

To test the proposed detrending algorithm for estimating the baseline wandering in the ECG signal, European ST-T database [21] is used. This data has $250 \mathrm{~Hz}$ sampling frequency with the acquisition time in one minute. The value of regularization parameter, $\lambda$ is chosen to be 2102.7757 in order to set the cut-off frequency with $1 \mathrm{~Hz}$. Figure 3 shows the result of removing the baseline fluctuation in the ECG signal using the improved detrending method. In order to verify the performance of the proposed detrending method, baseline wandering in the ECG signal is also removed using a $4^{\text {th }}$-order Butterworth high-pass filter (cutoff frequency $=1 \mathrm{~Hz}$ ) as shown in Figure 4.

Figures 3 and 4 demonstrate that detrending method and Butterworth filter can effectively cancel the baseline wandering. However, unlike the detrending method, a Butterworth high-pass digital filter deforms the morphological characteristic especially in the initial part of the ECG signal. In other words, detrending method is more effective in removing the baseline wandering and simultaneously maintaining the original characteristics in the ECG signal. For the frequency spectrum analysis, Figures 5(a) and 5(b) show the results of applying Discrete Fourier Transform to the trends estimated by the improved detrending method and a Butterworth high-pass digital filter, respectively.

Note that the spectral leakage in the estimated trend occurs less when the detrending method is applied.

\section{Conclusion}




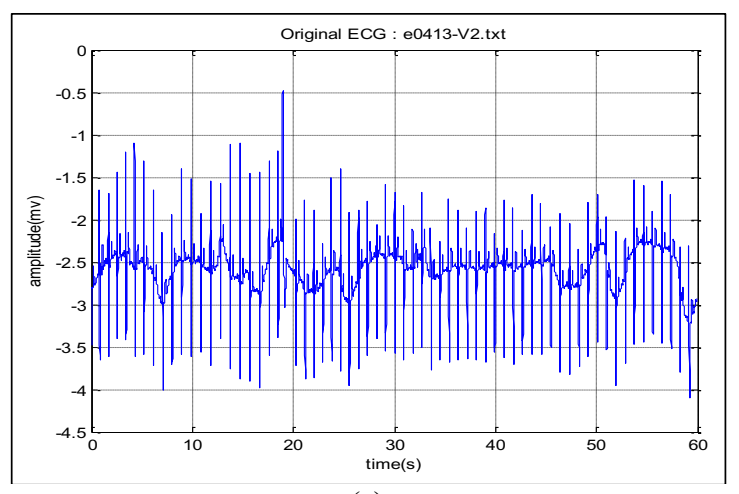

(a)
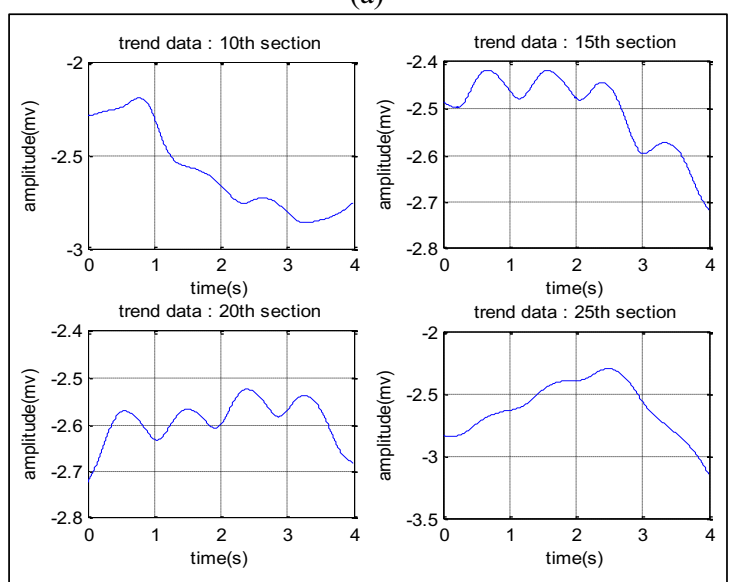

(c)

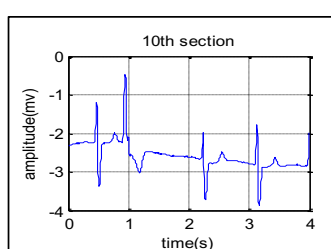
time(s)
20th section
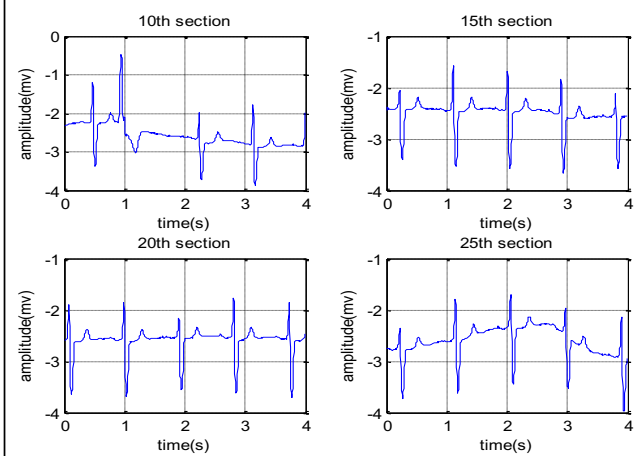

time $(s)$
25 th section

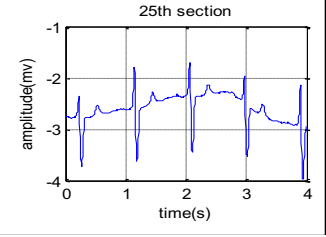

(b)
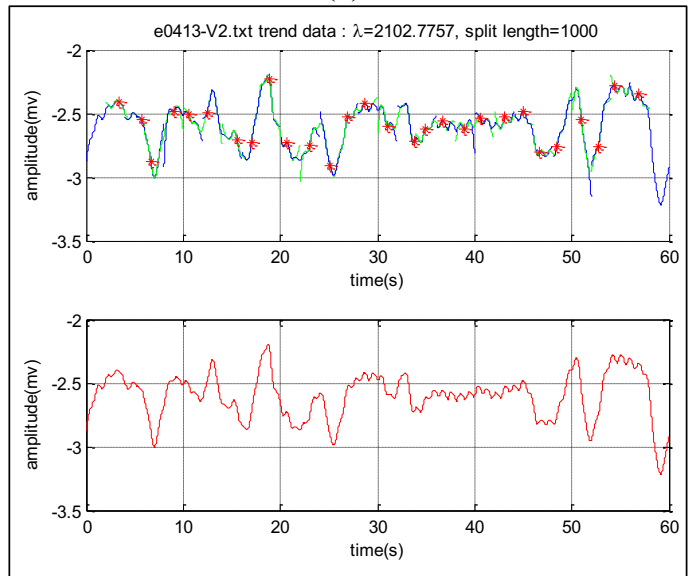

(d)

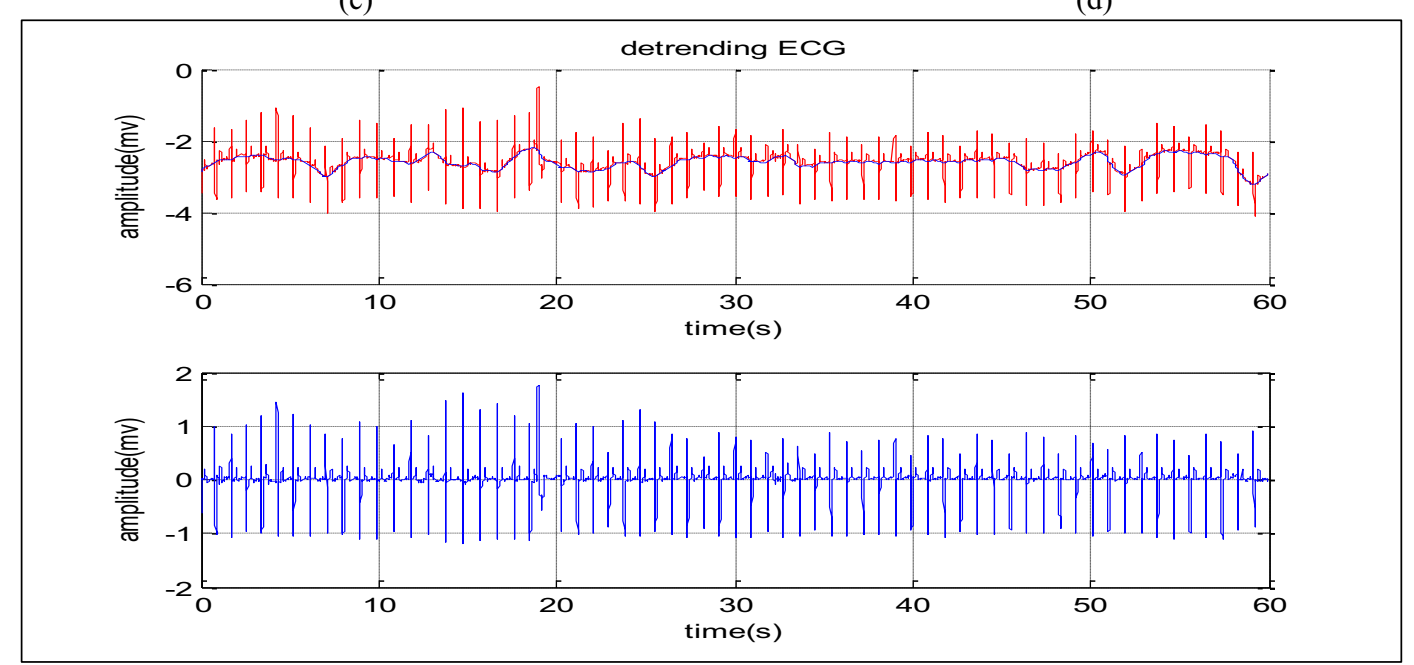

(e)

Fig. 3. The result of removing the baseline wandering in the ECG signal using the improved detrending method (a) the original ECG signal (b) the splited ECG-segments $(k=10,15,20,25)$ (c) the estimated local trends in the segments $(k=10$, $15,20,25)$ (d) the merging process of connecting local trends (e) the original ECG signal superimposed with the global trend in terms of baseline wandering (top) and the ECG signal with baseline wandering cancellation (below). 


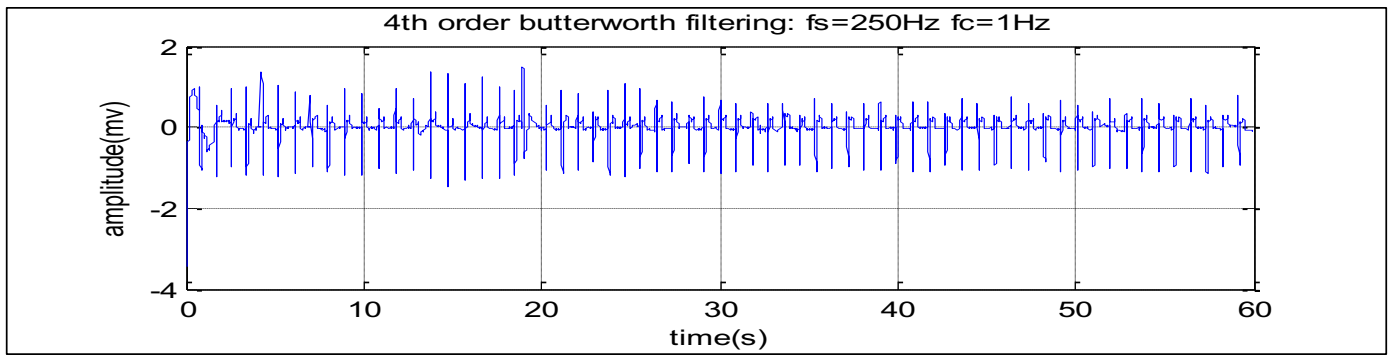

Fig. 4. The filtered ECG signal by $4^{\text {th }}$-order Butterworth high-pass filter.

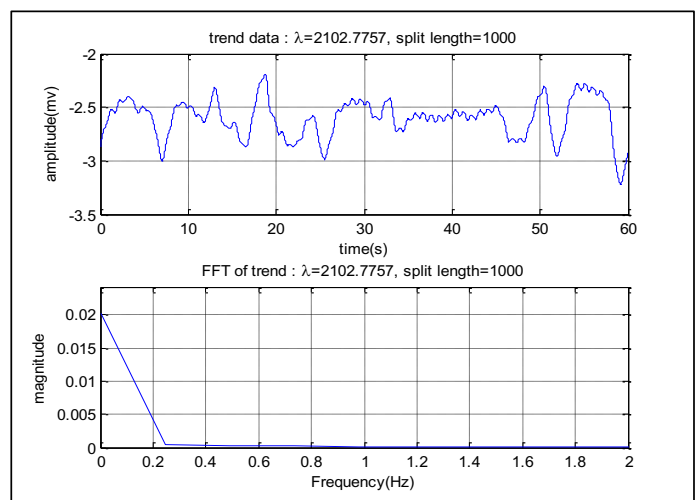

(a)

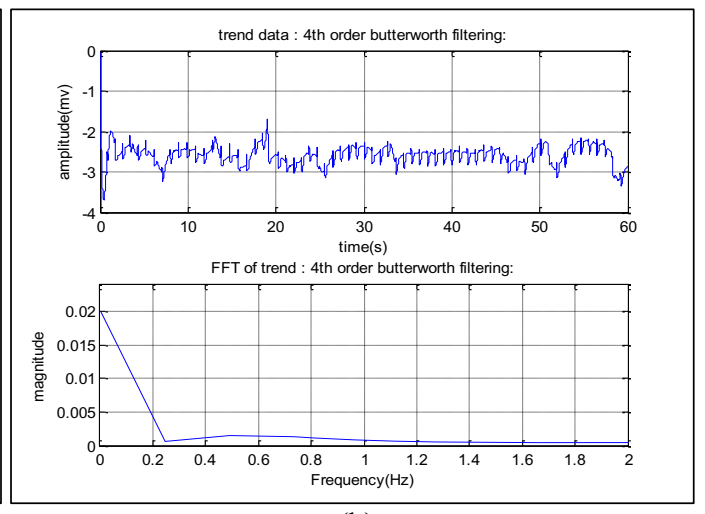

(b)

Fig. 5. Frequency spectrum of the trend estimated by (a) improved detrending method (b) the $4^{\text {th }}$-order Butterworth highpass filter.

The acquired ECG signal especially measured in a patient centric environment is vulnerable to the baseline wandering interference due to a patient's body moment or respiratory breathing. This lowfrequency noise may disturb the clinician in deciding the diagnostic parameters, thus methods are proposed to correct the baseline wandering. One common method is high-pass filtering but it may deform the important morphology of the ECG signal and evoke time delay. It should be also noted that a certain amount of spectral leakage above $1 \mathrm{~Hz}$ exists in the trend signal estimated by the conventional Butterworth high-pass filter (see Figure 5) and this leakage might cause the problem of deforming the diagnostic features in the ECG signal. The detrending method can be adopted to extract the global non-stationary trend in the ECG signal but it requires a large amount of matrix-inversion computations. To overcome this difficulty, a new detrending method is suggested by finding the local trends and merging them into a global one. The simulation results show that the proposed method can not only eliminate the baseline wandering but also keep the original waveform characteristics in the ECG signal. Thus, it can be concluded that our improved detrending method can effectively eliminate the baseline wandering without distorting morphological features in the original ECG signal.

\section{Acknowledgments}

This research was supported by Basic Science Research Program through the National Research Foundation of Korea (NRF) funded by the Ministry of Education, Science and Technology (NRF2013R1A1A2012393). 


\section{References}

[1] J.A. Van Alste and T.S. Schilder, Removal of base-line wander and power-line interference from the ECG by an efficient FIR filter with a reduced number of taps, IEEE Transaction on Biomedical Engineering 32 (1985), 1052-1060.

[2] B.N. Ezenwa, M.M. Gupta, P.N. Nikforuk and K. Pasad, A baseline tracking algorithm for drift reduction in electrocardiography, IEEE Engineering in Medicine and Biology Society 3 (1988), 1230-1232.

[3] P. Laguna, R. Jane and P. Caminal, Adaptive filtering of ECG baseline wander, IEEE Engineering in Medicine and Biology Society 2 (1992), 508-509.

[4] R. Jane, P. Laguna, N.V. Thakor and P. Caminal, Adaptive baseline wander removal in the ECG: Comparative analysis with cubic spline technique, Proceedings of Computers in Cardiology, Durham, North Carolina, 1992, pp. 143-146.

[5] S.V. Pandit, ECG baseline drift removal through STFT, IEEE Engineering in Medicine and Biology Society 4 (1996), $1405-1406$.

[6] V. Shusterman, S.I. Shah, A. Beigel and K.P. Anderson, Enhancing the precision of ECG baseline correction: Selective filtering and removal of residual error, Computers and Biomedical Research 33 (2000), 144-160.

[7] I. Dotsinsky and T. Stoyanov, Optimization of bi-directional digital filtering for drift suppression in electrocardiogram signals, Journal of Medical Engineering \& Technology 28 (2004), 178-180.

[8] M.A. Mneimneh, E.E. Yaz, M.T. Johnson and R.J. Povinelli, An adaptive kalman filter for removing baseline wandering in ECG signals, Computers in Cardiology, Valencia, 2006, pp. 253-256.

[9] J. Mateo, C. Sanchez, C. Vaya, R. Cervigon and J.J. Rieta, A new adaptive approach to remove baseline wander from ECG recordings using madaline structure, Computers in Cardiology, Durham, North Carolina, 2007, pp. 533-536.

[10] Z.D. Zhao and Y.Q Chen, A new method for removal of baseline wander and power line interference in ECG signals, International Conference on Machine Learning and Cybernetics, Dalian, China, 2006, pp. 4342-4347.

[11] Z.D. Zhao and L. Juan, Baseline wander removal of ECG signals using empirical mode decomposition and adaptive filter, International Conference on Bioinformatics and Biomedical Engineering, Chengdu, 2010, pp. 1-3.

[12] M. Blanco-Velasco, B Weng and K.E. Barner, ECG signal denoising and baseline wander correction based on the empirical mode decomposition, Computers in Biology and Medicine 38 (2008), 1-13.

[13] I.T. Iliev, S.D. Tabakov and V.T. Krasteva, Combined high-pass and power-line interference rejecter filter for ECG signal processing, Electronics, Sozopol, Bulgaria, 2008, pp. 49-54.

[14] M.Z.U. Rahman, R.A. Shaik and D.V.R.K. Reddy, Baseline wander and power line interference elimination from cardiac signals using error nonlinearity LMS algorithm, International Conference on Systems in Medicine and Biology, Kharagpur, 2010, pp. 217-220.

[15] K. Manivel and R.S. Rabindran, Noise removal for baseline wander and power line in electrocardiograph signals, International Journal of Advanced Research in Electrical, Electronics and Instrumentation Engineering 4 (2015), 11141122.

[16] M.A. Tinati and B. Mozaffary, A wavelet packets approach to electrocardiograph baseline drift cancellation, International Journal of Biomedical Imaging 2006 (2006), 1-9.

[17] Y. Luo, R.H. Hargraves, A. Belle, O. Bai, X. Qi, K.R. Ward, M.P. Pfattenberger and K. Najarian, A hierarchical method for removal of baseline drift from biomedical signals: Application in ECG analysis, The Scientific World Journal 2013 (2013), 1-10

[18] A. Fasano and V. Villani, Baseline wander removal for bioelectrical signals by quadratic variation reduction, Signal Processing 99 (2014), 48-57.

[19] S. Agrawal and A. Gupta, Fractal and EMD based removal of baseline wander and powerline interference from ECG signals, Computers in Biology and Medicine 43 (2013), 1889-1899.

[20] M.P. Tarvainen, P.O. Ranta-aho and P.A. Karjalainen, An advanced detrending method with application to HRV analysis, IEEE Transaction on Biomedical Engineering 49 (2002), 172-175.

[21] A.L. Goldberger, L.A.N. Amaral, L. Glass, J.M. Hausdorff, P.C. Ivanov, R.G. Mark, J.E. Mietus, G.B. Moody, B.K. Peng and H.E. Stanley, Physiobank, physiotoolkit, and physionet: Components of a new research resource for complex physiologic signals, Circulation 101 (2000), e215-e220. 